\title{
Trimeric Photosystem I facilitates energy transfer from phycobilisomes in Synechocystis PCC 6803
}

\author{
Parveen Akhtar ${ }^{1}$, Avratanu Biswas ${ }^{1,2}$, Fanny Balog-Vig ${ }^{1}$, Ildikó Domonkos ${ }^{1}$, László \\ Kovács ${ }^{1}$, Petar H. Lambrev ${ }^{1}$
}

${ }^{1}$ Biological Research Centre, Szeged, Institute of Plant Biology, Temesvári krt. 62, Szeged 6726, Hungary

\section{${ }^{2}$ Doctoral School of Biology, University of Szeged, Közép Fasor 52, Szeged 6726, Hungary}

\section{Short Title}

Trimeric Photosystem I facilitates energy transfer

\section{Author for contact}

\section{Petar H. Lambrev, lambrev.petar@brc.hu}

\section{Author Contributions}

P.A. and P.H.L. conceived the project and designed the experiments. P.A., A.B., and F.B.-V. prepared the biological samples and conducted most of the spectroscopic measurements. L.K. and P.A. performed P $_{700}$ oxidation measurements; I.D. and F.B.-V. did protein gel electrophoresis. P.A., A.B. and P.H.L. performed data analysis. P.A., A.B., and P.H.L. wrote the paper with contributions from all authors. P.H.L. is responsible for communication.

\section{Funding information}

National Research, Development and Innovation Office (grants FK-139067 to P.A. and 2018-1.2.1NKP-2018-00009 to P.H.L.), the Hungarian Ministry for National Economy (GINOP-2.3.2-15-201600058 to I.D.) and the Eötvös Loránd Research Network (SA-76/2021 to P.A.).

\section{One-sentence summary}

Cyanobacterial mutants with monomeric PSI have higher abundance of smaller phycobilisomes transferring more energy to PSII than to PSI, thus trimeric PSI is important for balanced energy flow.

\section{ABSTRACT}

In cyanobacteria, phycobilisomes serve as peripheral light-harvesting complexes of the two photosystems, extending their antenna size and the wavelength range of photons available for photosynthesis. The abundance of phycobilisomes, the number of phycobiliproteins they contain, and their light-harvesting function are dynamically adjusted in response to the physiological conditions. Phycobilisomes are also thought to be involved in state transitions that maintain the excitation balance between the two photosystems. Unlike its eukaryotic counterpart, PSI is trimeric in many cyanobacterial 
species and the physiological significance of this is not well understood. Here we compared the composition and light-harvesting function of phycobilisomes in cells of Synechocystis PCC 6803, which has primarily trimeric PSI, and the $\triangle p s a L$ mutant unable to form trimers. We also investigated a mutant additionally lacking the PsaJ and PsaF subunits of PSI, as PsaF has been proposed to facilitate interaction with phycobilisomes. Both strains with monomeric PSI accumulated significantly less phycocyanin (which constitutes the phycobilisome rods) per chlorophyll, while the allophycocyanin content was unchanged compared to WT. These data show that cells with monomeric PSI have higher abundance of smaller phycobilisomes. Steady-state and time-resolved fluorescence spectroscopy at room temperature and $77 \mathrm{~K}$ revealed that PSII receives more energy from the phycobilisomes at the expense of PSI in cells with monomeric PSI, regardless of the presence of PsaF. Taken together, these results show that the trimeric organization of PSI is advantageous for efficient and balanced excitation energy transfer from phycobilisomes in Synechocystis.

\section{INTRODUCTION}

Although the photosynthetic apparatus of cyanobacteria is largely similar to that of eukaryotic algae and plants, cyanobacteria are distinct in several key aspects. Cyanobacteria lack the membrane-intrinsic antenna proteins of the LHC family but utilize membrane-peripheral phycobilisomes (PBSs) to increase the absorption cross-section of the two photosystems, PSI and PSII. The organization and function of the cyanobacterial thylakoid membranes is defined by the large PBSs attached to them and the coexistence of the photosynthetic and respiratory electron-transport chains sharing electron carriers. Apart from their peripheral antenna systems, cyanobacterial and plant PSI differ by their quaternary structure and subunit composition. Four small hydrophobic protein subunits - PsaF, PsaJ, PsaK and PsaX (only present in thermophilic species) cover the membrane-exposed surface of cyanobacterial PSI (Fromme et al., 2003). The functional role of PsaF in cyanobacterial PSI, apart from structural stabilization, is not established. It has been suggested that PsaF may be involved in the docking of the PBSs (Hippler et al., 1999) and, under iron deficiency, to mediate the interaction of the PSI core with the peripheral antenna IsiA (Fromme et al., 2003; Akita et al., 2020). Whereas eukaryotic PSI is monomeric, in cyanobacteria it is found in the form of trimers and in some species tetramers. The PsaL subunit is crucial for trimer formation and mutants lacking PsaL only accumulate PSI monomers (Chitnis and Chitnis, 1993). The physiological advantages of PSI oligomerization in cyanobacteria are not clear. PSI trimers have higher far-red absorption thanks to the presence of long-wavelength chlorophylls (Chls) and it may facilitate quenching of excess excitation energy by the oxidized reaction center (RC) and help protect against photoinhibition and ROS generation (Karapetyan et al., 1999; Kłodawska et al., 2020). It has also been proposed that the trimeric state could facilitate excitation energy transfer (EET) from the PBS to PSI (Şener et al., 2004). 
The PBS is composed of phycobiliproteins (PBPs) and linker proteins organized as rods radiating from a membrane-attached core (MacColl, 1998; Arteni et al., 2009; Zheng et al., 2021). In the cyanobacterium Synechocystis PCC 6803 (hereafter called Synechocystis), six PBS rods connect three hexameric phycocyanin (PC) discs each and the core consists of three cylinders, each with two stacked allophycocyanin (APC) hexamers (Arteni et al., 2009). The ApcD and ApcE ( $\left.\mathrm{L}_{\mathrm{CM}}\right)$ polypeptides of the core are crucial for the interaction with the photosystems and contain the longest-wavelength $(680 \mathrm{~nm})$ 'terminal emitter' pigments of the PBS that transfer energy to Chls in both photosystems (Ashby and Mullineaux, 1999; Rakhimberdieva et al., 2001; Liu and Blankenship, 2019). In situ cryoelectron tomography has revealed the ordered arrays of PBS-PSII supercomplexes, where energy can presumably migrate also laterally between PBS making for a very efficient light-harvesting system (Rast et al., 2019; Li et al., 2021). Plausible routes for energy migration from PBS to PSI can be directly via interaction between them (Mullineaux, 1994; Liu et al., 2013) or indirectly via "spillover" from PSII to PSI (McConnell et al., 2002; Ueno et al., 2017). ApcE is responsible for EET to PSII, whereas ApcD is proposed to serve as an energy donor primarily for PSI (Ashby and Mullineaux, 1999; Dong et al., 2009; Liu and Blankenship, 2019).

The relative excitation of PSI and PSII can be rapidly regulated by the mechanism of state transitions, which is triggered by the redox state of the PQ pool (for reviews, see Mullineaux and Emlyn-Jones, 2005; Calzadilla and Kirilovsky, 2020). Several mutations in the PBS core are known to block or reduced the ability to perform state transitions, at least in some species (Ashby and Mullineaux, 1999; Dong et al., 2009; Calzadilla et al., 2019; Zlenko et al., 2019), highlighting the key role of the PBS in the process. However, the exact mechanism of cyanobacterial state transitions is under debate and alternative models are proposed, including a mobile PBS shuttling between PSI and PSII, regulated spillover (PSII-PSI energy transfer), and PSII quenching.

Cyanobacterial cells can modify the characteristics and abundance of PBSs in response to changes in the environmental conditions. Shortening of the PC rods under high growth light has been reported in several species, whereas low-intensity light elevates the PC content (Raps et al., 1985; Samson et al., 1994; Nomsawai et al., 1999). Macronutrient limitation results in extensive PBS breakdown (Salomon et al., 2013) that could provide the cell with amino acids - thereby, PBPs have obtained a secondary role as intracellular storage compounds.

Changes in the PBS can in turn affect the relative abundance of the membrane pigment-protein complexes. Genetically manipulated strains with shorter PBS rods or devoid of PBS accumulate more PSII relative to PSI (Nagarajan et al., 2014; Liberton et al., 2017). The PSI:PSII ratio in cyanobacteria is typically $2-4$ and varies depending on the light intensity and quality that the organism is cultured in (Murakami and Fujita, 1991). Low growth irradiance increases the abundance of PSI and vice versa. The sensing mechanisms controlling the PBPs and photosystem abundance are not well elucidated (Stadnichuk et al., 2015). Different growth conditions, such as light, temperature and available nutrients 
may change the PSI oligomeric state and the ratio of monomers to trimers (Ivanov et al., 2006; Salomon and Keren, 2011; Kłodawska et al., 2015). Furthermore, mutants unable to form PSI trimers have shown changes in the relative fluorescence emission of PBS components suggesting that the oligomeric state influences the PBS composition (Kłodawska et al., 2020).

In this work, we take a look into the relationship between the oligomeric state of PSI, the PBS composition and abundance, and the EET from PBS to the photosystems, by comparing Synechocystis (which has predominantly trimeric PSI) and the $\Delta p s a L$ mutant (unable to form trimers). In addition, we investigated a mutant lacking also the $\mathrm{F}$ and $\mathrm{J}$ subunits $(\Delta \mathrm{FIJL})$ to test if the PsaF and PsaJ subunits have specific role in EET from the PBS to PSI. We compare the PSI and PSII ratios as well as the PBP (PC and APC) content of the mutant cells with WT and will show that mutants with monomeric PSI have an altered composition and abundance of PBS. We also employed steady-state and time-resolved fluorescence spectroscopy at ambient and cryogenic temperature to evaluate the EET from PBS to the photosystems. By low-temperature time-resolved fluorescence spectroscopy we could separate PSII and PSI emission components and as well as populations of free, weakly, and strongly coupled PBPs. We show that the changes in the PBS composition affect the dynamics of EET in the cells and the excitation distribution between PSII and PSI, supporting the idea that PBS transfer energy more efficiently to trimeric than to monomeric PSI.

\section{MATERIALS AND METHODS}

\section{Cyanobacterial cultures and preparations}

Cyanobacterial cultures (Synechocystis sp. PCC 6803 wildtype (WT), $\Delta p s a L$, and $\Delta$ FIJL), thylakoid membranes, and isolated PSI complexes were prepared as described in Akhtar et al. (2021).

PBSs were prepared according to the protocol described in Garnier et al. (1994) with some adjustments. Briefly, photoautotrophically grown cells were centrifuged to pellet at $6000 \mathrm{~g}$ at $25^{\circ} \mathrm{C}$. The pellet was washed twice with phosphate buffer $(0.75 \mathrm{M}$ phosphate buffer, $1 \mathrm{mM}$ benzamidine hydrochloride hydrate, $1 \mathrm{mM}$ EDTA, pH 7.0 and $1 \mathrm{mM}$ of phenylmethylsulfonyl fluoride). The pellet was collected and treated with $0.2 \%$ of lysozyme and incubated for 1 hour in dark at $37^{\circ} \mathrm{C}$ with continuous shaking at $200 \mathrm{rpm}$. After incubation cells were pelleted down by centrifugation at $6000 \mathrm{~g}$ for 7 minutes, $14{ }^{\circ} \mathrm{C}$ and washed twice in phosphate buffer to remove the remaining lysozyme. The cells were then broken with glass beads ( $\leq 106 \mu \mathrm{m}$ diameter) using a homogenizer (Precellys Evolution) equipped with dry ice cooling compartment. The remaining glass beads were removed by centrifugation at $3000 \mathrm{~g}$ for $5 \mathrm{~min}$ at $14{ }^{\circ} \mathrm{C}$. The supernatant was then treated with $3 \%$ Triton-X100 with continuous stirring for $30 \mathrm{~min}$ at room temperature in dark and centrifuged at $21,000 \mathrm{~g}$ for $30 \mathrm{~min}$ to remove the unsolubilized material. The appropriate sample fraction was collected and loaded onto a sucrose density step gradient $(0.25$, $0.5,0.75,1 \mathrm{M}$ ) and centrifuged for $16 \mathrm{~h}$ at $104,000 \mathrm{~g} 14{ }^{\circ} \mathrm{C}$ for further purification. The gradient fraction containing PBSs was collected. 


\section{Pigment analysis}

138 Chls were extracted from the cell suspensions in 90\% methanol and the Chl contents were determined

139 spectrophotometrically using molar absorption coefficients described in Lichtenthaler (1987).

140 The phycobiliprotein content was determined as described in Zavřel et al. (2018). For PBPs isolation,

$141 \quad 100 \mathrm{ml}$ of the cells from each type were pelleted by centrifugation at $6000 \mathrm{~g}$ for $5 \mathrm{~min}$ and resuspend in 142 phosphate buffer (50 mM, pH 6.5) to total vol of $5 \mathrm{ml}$. The cells were broken using a Precellys Evolution 143 homogenizer with dry-ice-cooled chamber (10 cycles of braking, $30 \mathrm{~s}$ vortex, $5500 \mathrm{rpm}$ and two min cooling). The homogenate was then sonicated intermittently (five sec sonication with interval of $10 \mathrm{sec}$ rest, four times) by ultrasonicator at ice water temperature. Unbroken cells and cell debris were removed 146 by low-speed centrifugation. Cell homogenates were then ultracentrifuged at $104000 \mathrm{~g}$ for $60 \mathrm{~min}$. Absorption spectra of the transparent supernatant in the range of $220-750 \mathrm{~nm}$ were recorded to determine the soluble PBP content of the cells.

\section{Quantification of PSI and PSII}

150 The PSI and PSII concentrations were determined spectrophotometrically using the protocol described by Fujita and Murakami (1987). For $\mathrm{P}_{700}$ measurements the samples were suspended to $20 \mu \mathrm{g} / \mathrm{ml}$ (or optical density of 2 at $680 \mathrm{~nm}$ ) and for cytochrome (Cyt) to $60 \mu \mathrm{g} / \mathrm{ml}$ (or optical density of 6 at $680 \mathrm{~nm}$ ) in a buffer containing $20 \mathrm{mM}$ MES/NaOH, $\mathrm{pH}$ 6.4, $10 \mathrm{mM} \mathrm{MgCl}_{2}, 10 \mathrm{mM} \mathrm{CaCl}_{2}$. The control absorption spectra in the range of 350-750 $\mathrm{nm}$ were recorded from each sample. To estimate the concentration of $\mathrm{P}_{700}$ and Cyts, absorption spectra were recorded in the range 650-750 and 500-600 nm, respectively, with bandwidth of $1 \mathrm{~nm}$. The $\mathrm{P}_{700}$ was first oxidized with $1 \mathrm{mM}$ potassium ferricyanide and then reduced with sodium ascorbate. The difference spectra $(690-720 \mathrm{~nm})$ between oxidized and reduced $\mathrm{P}_{700}$ were identical to $\mathrm{P}_{700}$ and the difference at $700 \mathrm{~nm}$ was taken as the signal of $\mathrm{P}_{700} . \mathrm{P}_{700}$ abundance was estimated from the absorption difference with a molar extinct coefficient $\Delta \varepsilon_{\text {ox-red }}=64 \mathrm{mM}^{-1} \mathrm{~cm}^{-1}$ at 700 $\mathrm{nm}$. For PSII determination, first all Cyts were oxidized with $1 \mathrm{mM}$ of potassium ferricyanide. Then few grains of hydroquinone were added, followed by addition of sodium ascorbate and sodium dithionite. The difference spectra $(520-580 \mathrm{~nm})$ between hydroquinone-reduced and ascorbate-reduced had peak at $559 \mathrm{~nm}-$ Cyt $b_{559}$.

\section{Redox kinetics of P700}

The functional antenna size of PSI was estimated by the rate of light induced oxidation of $\mathrm{P}_{700} \mathrm{RC}$ under light limiting conditions. The oxidation kinetics of $\mathrm{P}_{700}$ upon illumination was followed by the measurement of absorbance change at $830 \mathrm{~nm}$ using Dual-PAM $100 \mathrm{Chl}$ a fluorometer (Walz,

168 Germany). Prior to measurement samples were dark-adapted for 3 minutes in the presence of $100 \mu \mathrm{M}$ 169 methylviologen (MV) and $20 \mu \mathrm{M}$ DCMU (3-(3,4-dichlorophenyl)-1,1-dimethylurea) then cells 170 equivalent to $20 \mu \mathrm{g} \mathrm{Chl}$ were filtered onto a $25 \mathrm{~mm}$ diameter glass fibre syringe filter disc (Whatman GF/C). The filter discs, placed between two microscopy slides with a spacer, were inserted between the 
172 fibre optics of the emitter-detector unit. Samples were illuminated with 5-s long 635-nm pulses at 173 various intensities $\left(6,31,140,251\right.$, and $805 \mu \mathrm{mol}$ photon $\left.\mathrm{m}^{-2} \mathrm{~s}^{-1}\right)$ consecutively and the oxidation

174 kinetics were recorded at a millisecond sampling rate.

\section{Steady-state absorption and fluorescence spectroscopy}

176 Absorption spectra in the range of 350-750 nm were recorded at room temperature with a Thermo

177 Evolution 500 dual-beam spectrophotometer. The measurements were performed in a standard glass cell

178 of $1-\mathrm{cm}$ optical path length with $1 \mathrm{~nm}$ spectral bandwidth.

179 Fluorescence emission spectra in the visible range were measured from the same samples at room 180 temperature and 77K on a FP-8500 (Jasco, Japan) spectrofluorometer. The sample were diluted to absorbance of 0.1 per $\mathrm{cm}$ at the red maximum. Emission spectra in the range of 620-780 $\mathrm{nm}$ were recorded with excitation wavelength of $440 \mathrm{~nm}$ and $580 \mathrm{~nm}$ and excitation/emission bandwidth of $3 \mathrm{~nm}$. The measurements were performed with $1 \mathrm{~nm}$ increment and $1 \mathrm{~s}$ integration time. For $77 \mathrm{~K}$ measurements samples were cooled in an optical cryostat (Optistat DN, Oxford Instruments, UK).

\section{Time-resolved fluorescence spectroscopy}

186 Picosecond time-resolved fluorescence measurements were performed with a time-correlated singlephoton counting instrument (FluoTime 200/PicoHarp 300 spectrometer, PicoQuant). Excitation was provided by Fianium WhiteLase Micro (NKT Photonics, UK) supercontinuum laser, generating whitelight pulses with a repetition rate of $20 \mathrm{MHz}$. Excitation wavelengths of 440 and $580 \mathrm{~nm}$ were used to excite selectively Chls and PBSs. The fluorescence decays were recorded at wavelengths of 600-744 $\mathrm{nm}$ with $8 \mathrm{~nm}$ steps, at room temperature, and $605-760 \mathrm{~nm}$ with $5 \mathrm{~nm}$ steps at $77 \mathrm{~K}$. All the samples were diluted to an absorbance of 0.03 at excitation wavelength. For the room temperature measurements, the suspension (whole cells or isolated complexes) was placed in $1 \mathrm{~mm}$ flow cell and circulated at a flow rate of $4 \mathrm{ml} / \mathrm{min}$. For $77 \mathrm{~K}$ measurements, the suspension was placed in a $1 \mathrm{~mm}$ demountable cryogenic quartz cell and cooled in an optical cryostat (Optistat DN, Oxford Instruments, UK). The total instrument response (IRF) measured using $1 \%$ Ludox as scattering solution has width of 40 ps. Global multiexponential lifetime analysis with IRF reconvolution was performed using MATLAB.

\section{RESULTS}

\section{Changes in the pigment stoichiometry}

We cultured Synechocystis (WT), which contains PSI trimers and the two mutants with monomeric PSI, $\Delta p s a L$ and $\Delta$ FIJL, under the exact same conditions, to examine the phenotype effects of the mutations. Both mutants appeared more greenish in colour suggesting a change in the pigment composition of the cells. Accordingly, absorption spectra of the supernatant obtained after sedimenting the broken cell debris (Supplementary Fig. S1) show a distinct shoulder around $650 \mathrm{~nm}$ in both mutants $-\triangle p s a L$ and 
cultures from absorption spectra of the supernatant (Table 1). The Chl content was measured from methanol extracts of either the cell debris or whole cell sediment, yielding approximately equal results. We found that the ratio of PC to Chl was unchanged between the WT and mutant cultures. However, the monomeric PSI mutants contained significantly higher amount of APC (up to 80\% more than in the WT), hence lower PC:APC and Chl:APC ratios. If we assume that all, or almost all, PC in the WT is found in the PBS rods, the lower PC:APC ratio means that the PBS in the mutants has either fewer or shorter PC rods. On the other hand, the more abundant APC indicates a higher number of PBS cores in the cells. Assuming a basic tricylindrical APC core architecture of the PBS, wherein each PBS contains 36 APC $\alpha \beta$ subunits, we can estimate the Chl:PBS ratio (Table 1). From these data it follows that both the composition and the number of the PBSs are altered in the mutants with monomeric PSI - they contain more PBSs as a whole (on Chl basis) but the PBSs are smaller, binding fewer PC units.

It must be noted that the PC:APC ratios in the WT are higher than expected. PBSs are typically found to contain six rods with three PC hexamers per tricylindrical core (Arteni et al., 2009; Rast et al., 2019), which amounts to a PC:APC ratio of 3:1. Although one could merely attribute the discrepancy to a systematic error in the PC:APC estimation, it is interesting to point out that we obtained different results from isolated PBS using the same measurement methodology (Supplementary Table S1). The PC:APC ratio of isolated PBS was found to be around 4:1 - similar to previous reports. More importantly, the ratios were the same in PBSs of the WT and mutants, as evident from their nearly identical absorption spectra (Supplementary Fig. S1). It must be concluded that WT cells under our growth conditions contain extra PC, which is either not connected to the PBS in vivo or (more likely) is weakly connected such that is dissociated during isolation of PBS.

Table 1. Pigment content of cell cultures

\begin{tabular}{|l|c|c|c|c|c|c|c|}
\hline Type & $\begin{array}{c}\text { PC } \\
(\mu \mathrm{mol} / \mathrm{L})\end{array}$ & $\begin{array}{c}\text { APC } \\
(\mu \mathrm{mol} / \mathrm{L})\end{array}$ & $\begin{array}{c}\text { Chl } \\
(\mu \mathrm{mol} / \mathrm{L})\end{array}$ & PC:APC & Chl:PC & Chl:APC & Chl:PBS \\
\hline WT & $2.0 \pm 0.4^{*}$ & $0.28 \pm 0.04$ & $10 \pm 1$ & $7.2 \pm 0.6$ & $5.1 \pm 0.3$ & $36 \pm 2$ & 1305 \\
\hline$\Delta p s a L$ & $2.2 \pm 0.3$ & $0.50 \pm 0.07$ & $11 \pm 1$ & $4.4 \pm 0.2$ & $4.9 \pm 0.3$ & $19 \pm 2$ & 767 \\
\hline$\Delta$ FIJL & $1.8 \pm 0.1$ & $0.37 \pm 0.14$ & $8 \pm 2$ & $5.1 \pm 0.3$ & $4.9 \pm 0.5$ & $24 \pm 4$ & 915 \\
\hline
\end{tabular}

* values are represented as the mean \pm standard error of four biological replicates

The striking change in the PBS content and composition found in the monomeric PSI mutants compared to WT Synechocystis prompted to test whether there was a corresponding change in the photosystem stoichiometry. To this end, we estimated the number of PSI and PSII RCs in the cells by the absorption difference of the oxidized and reduced forms of $\mathrm{P}_{700}$ and Cyt $b_{559}$, respectively (Table 2 and Supplementary Fig. S2). We found $\mathrm{P}_{700}$ :Cyt $b_{559}$ ratios of 1.5-1.7 that were not significantly different between mutants and WT. These values are similar to the ones reported by Murakami and Fujita (1991). 
PSI:PSII ratios were twice as high. A RC ratio of 1.5 means that there are equimolar ratios of PSI trimers $\left(\mathrm{PSI}_{3}\right)$ and PSII dimers $\left(\mathrm{PSII}_{2}\right)$ in WT or three monomeric PSI per $\mathrm{PSII}_{2}$ in the mutants. From these ratios and the number of Chls in PSI and PSII, we can estimate 3-4 PSI and the same number of PSII complexes per PBS in the WT. In the monomeric mutants, although the number of PBS is apparently increased, there are still more (monomeric) PSI complexes per PBS (6-7) but fewer PSII (2-3). Thus, we could potentially interpret the increased number of PBS in the mutants as an adaptive response that compensates for the number of PSI per PBS.

Table 2. Stoichiometric ratios of PSI, PSII and PBS

\begin{tabular}{|l|c|c|c|c|c|c|c|}
\hline Type & $\begin{array}{c}\mathrm{P}_{700}: \text { Cyt } \\
b_{559}\end{array}$ & PSI $_{3(1)}: \mathrm{PSII}_{2}$ & Chl:PSI $_{3(1)}$ & $\mathrm{P}_{700}: \mathrm{PBS}^{*}$ & $\begin{array}{c}\text { Cyt } \\
b_{559}: \mathrm{PBS}\end{array}$ & $\mathrm{PSI}_{3(1)}: \mathrm{PBS}$ & $\mathrm{PSII}_{2}: \mathrm{PBS}$ \\
\hline $\begin{array}{l}\mathrm{WT} \\
\left(\mathrm{PSI}_{3}\right)\end{array}$ & $1.6 \pm 0.2$ & 1.0 & 285 & 11.4 & 7.2 & 3.8 & 3.6 \\
\hline $\begin{array}{l}\Delta p s a L \\
\left(\mathrm{PSI}_{1}\right)\end{array}$ & $1.5 \pm 0.2$ & 3.0 & 92 & 6.3 & 4.0 & 6.3 & 2.0 \\
\hline $\begin{array}{l}\Delta \mathrm{FIJL} \\
\left(\mathrm{PSI}_{1}\right)\end{array}$ & $1.7 \pm 0.1$ & 3.1 & 88 & 7.8 & 5.0 & 7.8 & 2.5 \\
\hline
\end{tabular}

* assuming all PBS have a tricylindrical core with two APC hexamers per cylinder

\section{Steady-state fluorescence emission spectra}

As a further confirmation of the pigment stoichiometry changes, we recorded fluorescence emission spectra of intact WT, $\Delta p s a L$, and $\Delta$ FIJL cells at $77 \mathrm{~K}$ (Figure 1). The spectra recorded with $580 \mathrm{~nm}$ excitation (primarily absorbed by PC) have well-defined peaks at 650, 660, 687, and $720 \mathrm{~nm}$. The peaks at 650 and $660 \mathrm{~nm}$ correspond to PC and APC and the ones at 687 and $720 \mathrm{~nm}$ - to PSII and PSI, respectively. In accordance with the higher amount of PC determined in the WT, the spectra showed more intense emission at $650 \mathrm{~nm}$. The ratio of fluorescence emitted at $650 \mathrm{~nm}$ to $660 \mathrm{~nm}$ decreased in the monomeric PSI types in line with the decreased PC:APC ratio. Increased PC emission is also detectable in the room-temperature fluorescence spectra of the WT cells, albeit not as pronounced two monomeric PSI types, $\Delta p s a L$ and $\Delta$ FIJL. 

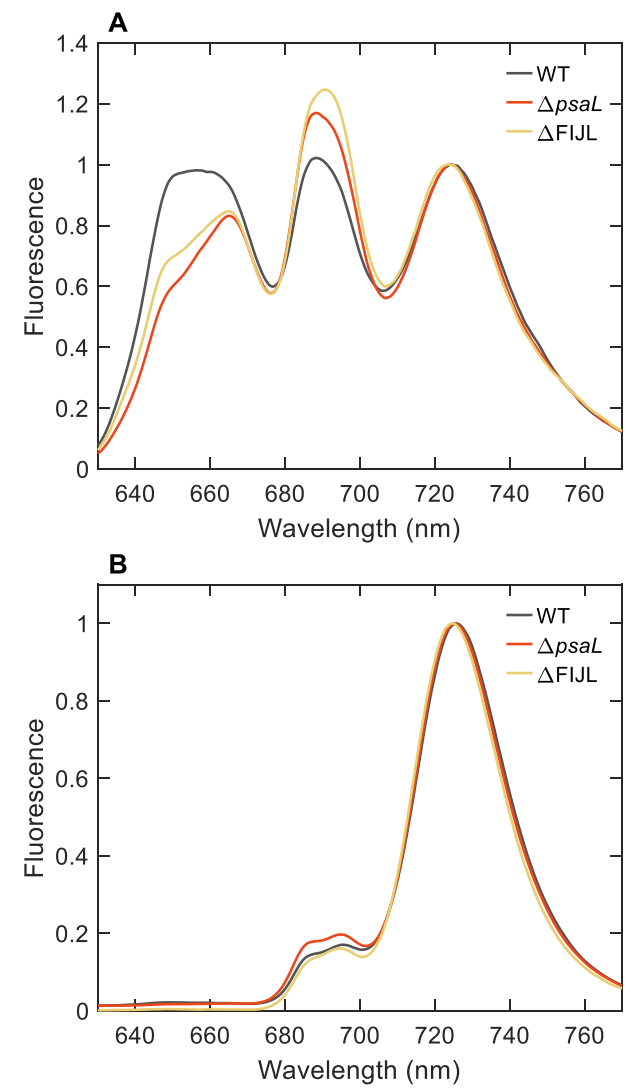

Figure 1. Fluorescence emission spectra of intact cells of Synechocystis WT, $\Delta p s a L$ and $\Delta$ FIJL recorded at $77 \mathrm{~K}$. (A) Excitation wavelength $580 \mathrm{~nm}$; (B) Excitation wavelength $440 \mathrm{~nm}$. The spectra are normalized at $720 \mathrm{~nm}$.

The photon energy absorbed by the PBSs is ultimately distributed between both PSI and PSII as evident from their corresponding emission peaks. The relative amplitudes of the 687 and $720 \mathrm{~nm}$ peaks suggest that in $\triangle p s a L$ and $\triangle \mathrm{FIJL}$ cells more energy is transferred to PSII compared to WT. In contrast, the fluorescence spectra recorded with $440 \mathrm{~nm}$ excitation (almost exclusively absorbed by the photosystems) show no significant difference in the ratio of the PSII and PSI peaks. This is expected because the PSI: PSII stoichiometric ratio (on monomer basis) is unchanged. Thus, the stronger PSII emission upon $580 \mathrm{~nm}$ indicates that the distribution of excitation energy from PBSs to PSII/PSI is altered in the monomeric PSI types.

\section{P700 oxidation kinetics}

To compare the effective antenna size of PSI, we recorded the oxidation kinetics of $\mathrm{P}_{700}$ (absorption transients at $830 \mathrm{~nm}$ ) in intact cells and isolated PSI complexes upon illumination in the presence of DCMU and MV. DCMU prevents re-reduction of $\mathrm{P}_{700}{ }^{+}$by electrons from PSII and MV accepts electrons from PSI keeping the acceptor side of PSI in oxidized state and minimizing cyclic electron flow. The oxidation curves at different light intensities are shown in Supplementary Fig. S4 and the oxidation rates obtained by fitting logistic or exponential kinetics to the curves are in Figure 2. At all light intensities in

274 the range 6-805 $\mu \mathrm{mol} \mathrm{m}^{-2} \mathrm{~s}^{-1}$, the oxidation rates in WT Synechocystis cells were higher than either of the monomeric PSI types - up to $60 \%$ at $140 \mu \mathrm{mol} \mathrm{m}^{-2} \mathrm{~s}^{-1}$. These data indicate that the effective antenna 
size of monomeric PSI in vivo is smaller compared to trimeric PSI. The oxidation kinetics were measured with $635 \mathrm{~nm}$ light, predominantly absorbed by the PBS. Hence, a possible explanation for the different rates could be that PBS transfer energy more effectively to trimeric than to monomeric PSI. This hypothesis is supported by the fact that the $\mathrm{P}_{700}$ oxidation rates were similar in monomeric and trimeric isolated PSI (Figure 2B) as well as in thylakoid membranes, which lack PBSs (Supplementary Fig. S5).
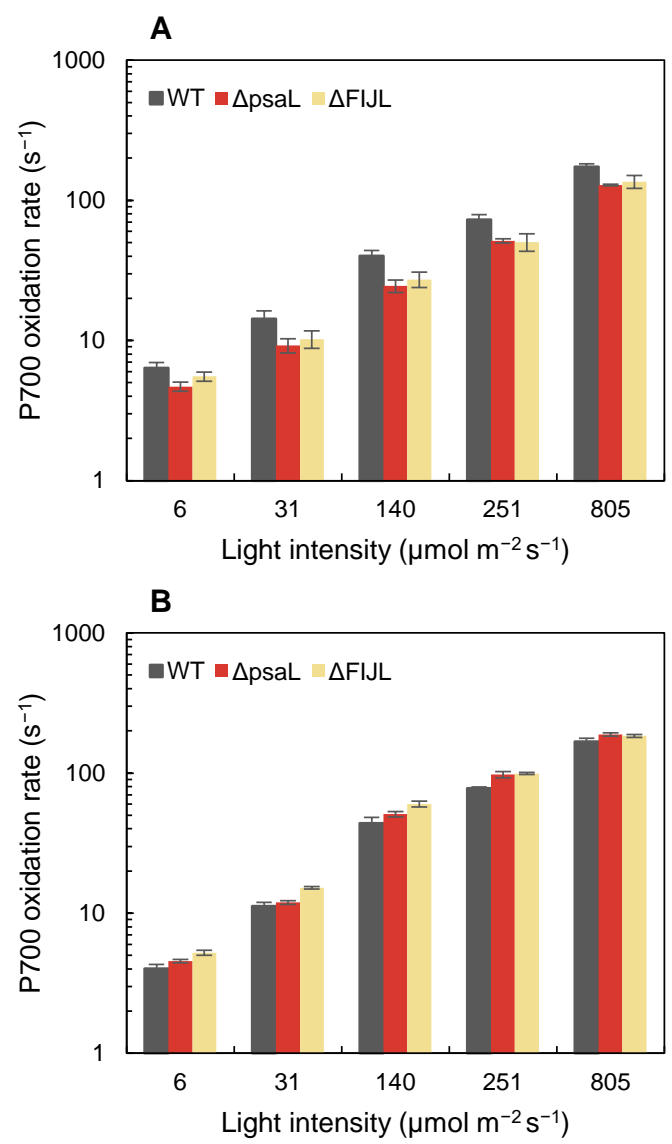

Figure 2. $\mathrm{P}_{700}$ oxidation rates. (A) intact cells of Synechocystis WT, $\Delta p s a L$ and $\Delta \mathrm{FIJL}$; (B) isolated PSI. The rates are calculated from the differential absorption at $830 \mathrm{~nm}$ induced by pulses of different light intensity, applying logistic or exponential fit (for intensities under and above $100 \mu \mathrm{mol} \mathrm{m} \mathrm{m}^{-2} \mathrm{~s}^{-1}$, respectively). Error bars indicate standard errors from four independent experiments. Note the logarithmic vertical scale.

\section{Fluorescence kinetics of cells at room temperature}

288 We employed picosecond time-resolve fluorescence spectroscopy to gain better understanding of the excitation energy partitioning in the intact cells of Synechocystis with trimeric (WT), monomeric $(\Delta p s a L)$ and subunit-depleted ( $\triangle \mathrm{FIJL})$ PSI. Time-resolved fluorescence enables better separation of emission components, for example emission of PBSs that are energetically coupled to the photosystems from free PBPs and can detect changes in the architecture and supramolecular organisation of the photosynthetic complexes that affect the EET. We applied global five-exponential analysis of the fluorescence decays recorded in the wavelength range $600-720 \mathrm{~nm}$ after excitation at $580 \mathrm{~nm}$. Figure 3 compares the lifetimes and decay-associated emission spectra (DAES) of WT, $\Delta p s a L$, and $\Delta$ FIJL cells. 
The lifetimes and spectra are very similar between genotypes and are comparable to previously published results on cyanobacterial cells (Mullineaux and Holzwarth, 1991; Tian et al., 2011; Akhtar et al., 2020). The first two components (Figure 3A,B), with respective lifetimes around $30 \mathrm{ps}$ and 90-100 ps, represent EET within the PBS (Akhtar et al., 2020). The DAES have characteristic positive and negative peaks around 650,660, and $680 \mathrm{~nm}$, signifying decay and rise of the emission from PC, APC and red-shifted $\mathrm{APC}\left(\mathrm{APC}_{680}\right)$, respectively. The third component with a lifetime about $170 \mathrm{ps}$ represents mainly decay of APC excitations in PBS coupled to photosystems and the fourth, around 500-600 is associated with trapping in PSII. A longer-lived component (1.4-1.6 ns, not plotted) indicated the presence of a negligible amount (2-3\%) of uncoupled PBPs.
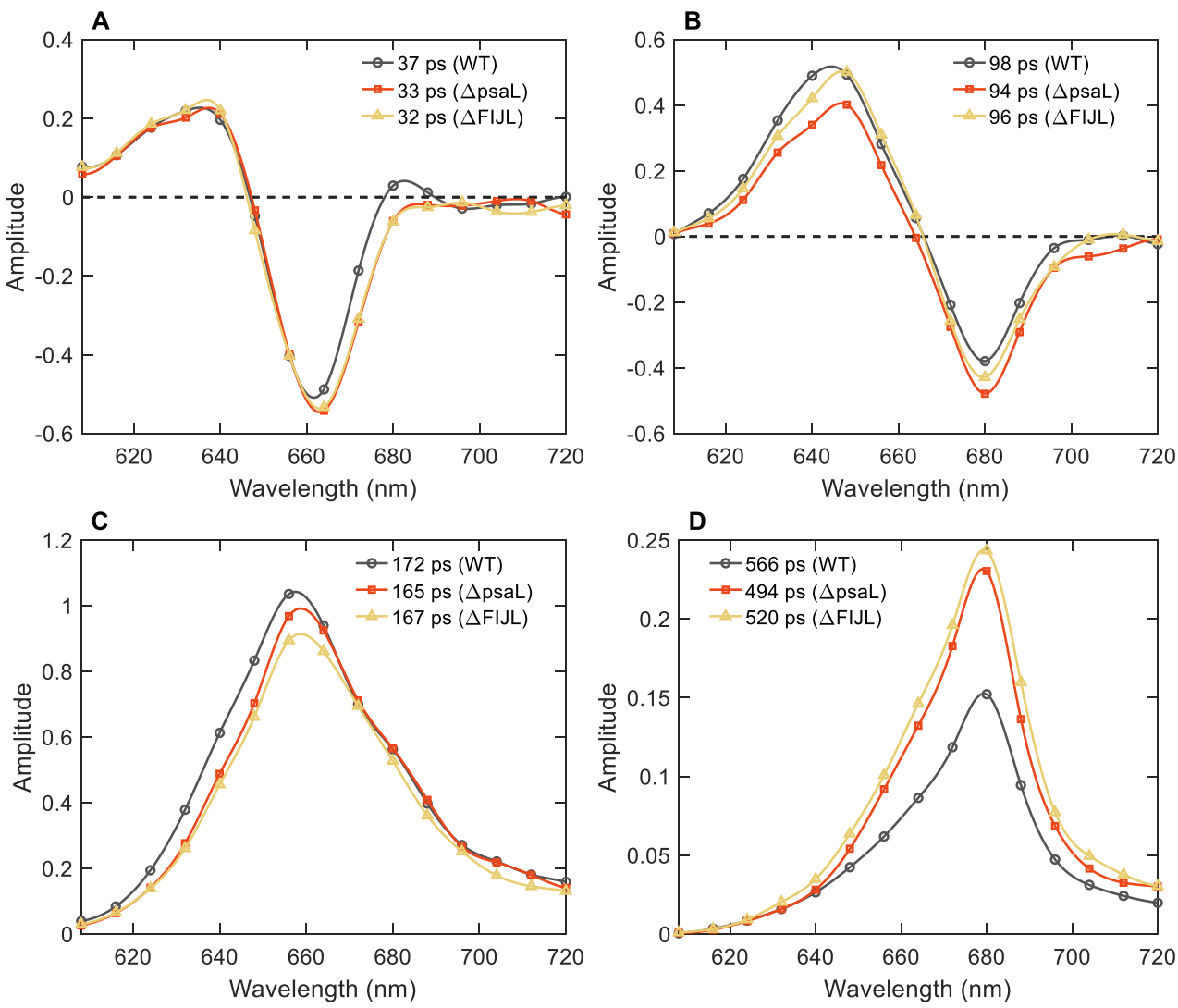

Figure 3. DAES of Synechocystis cells obtained by global lifetime analysis of the fluorescence decays recorded at room temperature with $580 \mathrm{~nm}$ excitation. Panels A-D compare individual DAES for WT (black circles), $\Delta p s a L$ (red squares), and $\triangle \mathrm{FIJL}$ (gold triangles) cells. The spectra are scaled to the total number of photons counted. The longest-lived (1.3-1.6 ns) component is omitted.

As seen in Figure 3, there is little difference between the genotypes as concerning the shape of the DAES. On close inspection, the DAES of WT cells display relatively larger amplitudes at $640-650 \mathrm{~nm}$ (Figure 3B,C), consistent with their higher PC:APC ratio. There was also a small but significant difference in the PBS-associated decay lifetimes - in WT we obtained $35 \pm 1,97 \pm 2$, and $172 \pm 3$ ps, compared to $31 \pm 1,90 \pm 2$, and $162 \pm 2 \mathrm{ps}$ in $\triangle p s a L$ (each value an average of four culture batches). This result is consistent with longer PC rods in the PBSs of WT cells, which slows down the rod-core equilibration dynamics (Sandström et al., 1988; Zhang et al., 1997). 
The most notable kinetic difference between WT cells and those with monomeric PSI is in the PSII decay component (Figure 3D). In the WT, the peak amplitude of this component was $15 \pm 2 \%$, whereas in either of the monomeric PSI types it was $20 \pm 2 \%$. This result confirms the finding that a larger proportion of excitation energy is transferred to PSII in cells with monomeric PSI, compared to cells with trimeric PSI. Moreover, as we do not observe significant amount of uncoupled PBSs in any of the Synechocystis cells, we can make the reverse conclusion - namely, that PBSs transfer more energy to PSI when it is trimeric rather than monomeric.

\section{Fluorescence kinetics of cells at $77 \mathrm{~K}$}

We further examined the fluorescence decay kinetics of intact cells at $77 \mathrm{~K}$ for better resolution of the different pigment groups. Most importantly, the emission from the long-wavelength "red" Chls in PSI is well pronounced allowing us direct comparison of the energy flow to the two photosystems. Global analysis of the fluorescence decays resulted in six decay lifetimes and DAES. Individual DAES for the three Synechocystis genotypes are compared in Figure 4. We will first briefly describe the kinetic components in WT cells. The fastest component (13 ps, Figure 4A) shows decay of PC emission at 640 $\mathrm{nm}$ and concomitant rise of APC emission at $660 \mathrm{~nm}$. In addition, EET from bulk to red Chls in PSI occurs on this timescale (690 to $720 \mathrm{~nm}$ ). The second component (56 ps, Figure 4B) shows decay of both PC and APC and rise of the red-shifted $\mathrm{APC}_{680}$. The 145-ps component (Figure 4C) has positive peaks corresponding to all three PBP groups ( $\mathrm{PC}, \mathrm{APC}$ and $\mathrm{APC}_{680}$ ) that evidently decay as energy is transferred to Chls. Also notable are the negative peaks at $690 \mathrm{~nm}$ (PSII) and $720 \mathrm{~nm}$ (PSI). The PSII and PSI emission components decay mainly with lifetimes of 373 ps and 982 ps (Figure 4D,E) while the final component (3.8 ns, Figure 4F) is of negligible amplitude. Remarkably, PC fluorescence detected at 640-650 nm spans the entire range of decay timescales (in WT). A sizeable fraction of longlived PC is less efficient in transferring excitation energy downstream (note the $650 \mathrm{~nm}$ peaks in Figure $4 \mathrm{C}-\mathrm{E})$. It is also of note that a considerable proportion of photon energy absorbed by the PBS is delivered to PSI, judging by the height of the DAES peaks around $720 \mathrm{~nm}$.

Compared to WT, the DAES of both monomeric PSI types showed notable differences. Remarkable are the kinetic differences in the $640-680 \mathrm{~nm}$ wavelength range reflecting PBPs emission. In $\triangle p s a L$ and $\triangle$ FIJL cells, PC emission decays faster than in WT - the peaks at $650 \mathrm{~nm}$ in the long-lived DAES all but vanish. Conversely, the amplitudes of the APC peaks at 660 and $680 \mathrm{~nm}$ are larger than in WT. Especially obvious is the larger negative peak at $680 \mathrm{~nm}$ in the 58-ps DAES (Figure 4B) and corresponding positive peak in the 136-ps DAES (Figure 4C). These results show unequivocally that the altered PBP stoichiometry results in changes in the excitation dynamics of the intact and energetically coupled PBSs.

The fluorescence kinetics at $77 \mathrm{~K}$ also unambiguously confirm that a larger proportion of PBS excitations end up on PSII when PSI is monomeric (compare the amplitudes of the 1-ns DAES at 695 $\mathrm{nm}$ relative to $725 \mathrm{~nm}$ in Figure 4E). 

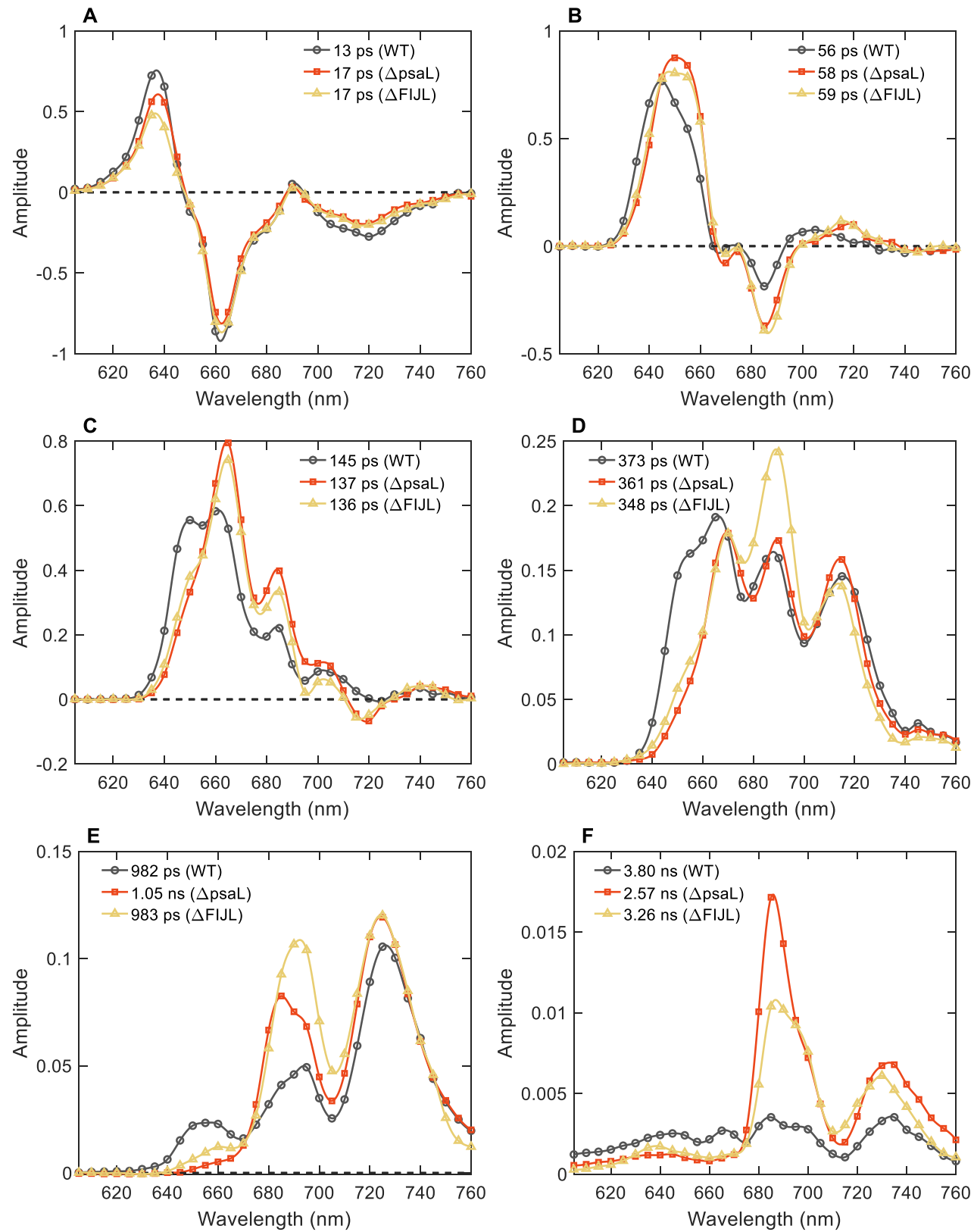

354 Figure 4. DAES of Synechocystis cells obtained by global lifetime analysis of the fluorescence decays recorded at $77 \mathrm{~K}$ with $580 \mathrm{~nm}$ excitation. Panels A-F compare individual DAES for WT (black circles), $\Delta p s a L$ (red squares), and $\triangle$ FIJL (gold triangles) cells. The spectra are scaled to the total number of photons counted.

357 As a control, we analysed the fluorescence kinetics at $77 \mathrm{~K}$ upon $440 \mathrm{~nm}$ excitation, which excludes the

358 PBS contribution to the dynamics. In this case, the lifetimes and DAES of intact cells were virtually

359 identical to the ones reported earlier for isolated thylakoid membranes (Supplementary Fig. S6). The

360 fluorescence decays reflect mainly the dynamics in PSI, as it has 4-5 times larger absorption cross section than PSII. The most significant difference in the fluorescence kinetics is the reduced amplitude of the DAES emitting at $710-715 \mathrm{~nm}$, attributed to the loss of red Chls at the trimerization region (Akhtar et al., 2021). 
Additionally, we compared the fluorescence kinetics of isolated PBSs of WT, $\Delta p s a L$, and $\Delta \mathrm{FIJL}$ at room temperature and $77 \mathrm{~K}$ (Supplementary Figs. S7, S8). Interestingly, the clear differences in the PC dynamics, that were observed in whole cells, could not be detected in the isolated PBS. This result is in line with the fact that changes in the PC:APC ratio were detected in whole cells but not in isolated PBS.

\section{DISCUSSION}

A key finding of this study is that the oligomeric state of PSI exerts control over the abundance of PBPs in Synechocystis and, likely, the architecture of the PBSs. The $\Delta p s a L$ and $\Delta$ FIJL genotypes with monomeric PSI contained more PBSs on Chl basis compared to the WT genotype with trimeric PSI but, at the same time, fewer PCs per PBS (Tables 1 and 2). In agreement with the spectroscopic quantification, semiquantitative Tricine-SDS-PAGE analysis showed lower PC:APC ratios in cells of the monomeric PSI mutants (Supplementary Fig. S9). Our results are in line with the changes in fluorescence spectra of the $\Delta p s a L$ mutant of Synechocystis, reported by Kłodawska et al. (2020) and attributed to differences in the PBS composition.

By comparing the data from cells and isolated PBSs we can conclude that under the present cultivation conditions WT Synechocystis cells contain weakly bound PCs that are dissociated upon PBS isolation. We cannot conclusively determine whether the additional PC makes up for longer rods radiating from the PBS core (the PC:APC ratio suggests six PC hexamers per rod) or more (laterally bound) rods. It is evident, however, that the higher PC content slows down energy migration in the PBS and consequently from the PBS to the photosystems. It has been demonstrated that the rod-core equilibration time increases with the length of the PC rods (Sandström et al., 1988; Zhang et al., 1997). Here, we have found that a fraction of the PC is exceedingly long-lived, suggesting weak energetic coupling that favours parallel or unspecific binding of PC rods. These "semi-free" rods may be easily detached during PBS isolation.

Remarkably, both genotypes with monomeric PSI, grown under the same conditions, contain less PC per PBS. We detected no long-lived PC population in the fluorescence kinetics and there is no evidence for loss of PC during biochemical isolation, which suggests that the PBSs have shorter strongly-coupled PC rods. The modulation of the size and number of PBSs can be understood as an adaptive response. Cyanobacteria are capable of acclimatory regulation of the PBS in response to the physiological conditions. Growth under high irradiance changes the abundance of PBS, reducing the cell PC content (Raps et al., 1985) and shortening of the PC rods has been reported in Synechococcus (Samson et al., 1994), S. maxima (Garnier et al., 1994), and S. platensis (Nomsawai et al., 1999). The rod length seems to be regulated by specific linker proteins (Nomsawai et al., 1999). We could not detect any differences in the PBPs or linker protein content of the PBSs (Supplementary Fig. S9), except for a reduced content of FNR in PBS of $\triangle$ FIJL. Interestingly, FNR was also missing from a mutant with truncated rods (Leganes et al., 2014). PBPs are also reservoirs for nutrients, degraded when nutrients, e.g. nitrogen, are 
scarce (Salomon et al., 2013), so it is plausible that downstream metabolism changes will also alter the PBP content.

We reason that the absence of trimeric PSI in Synechocystis results in suboptimal or imbalanced EET, consequently electron transfer, which causes a change in the PBS content and composition. The most logical explanation reconciling all our data is that EET from the PBS to PSI is less efficient in the $\Delta p s a L$ and $\triangle$ FIJL mutants than it is in WT. Results in support of this are the higher PSII:PSI emission ratio upon PBS excitation (but not upon Chl excitation) and the diminished effective PSI antenna size in $\Delta p s a L$ and $\Delta$ FIJL cells (but not thylakoid membranes or isolated PSI). As to why trimeric PSI might be more suited for accepting energy from the PBS, there could be several possible reasons. PSI trimers might have higher affinity to the PBSs forming a more stable PBS-PSI supercomplex. This may be in contrast to structural modelling that suggests the existence of a PBS fraction directly attached to PSI monomers (Zlenko et al., 2016). We must also consider the possible role of state transitions (Mullineaux and Emlyn-Jones, 2005; Calzadilla and Kirilovsky, 2020). The $\Delta p s a L$ mutant of Synechococcus PCC 7002 was found to be capable of performing a state II-state I transition faster than the wild type (Schluchter et al., 1996) and Aspinwall et al. (2004) concluded that this was because of higher rates of PBS diffusion. Notably, if the PBSs in $\triangle p s a L$ are indeed smaller, this should in itself contribute to their higher mobility (Sarcina et al., 2001). However, there is no indication that the mutant is locked or preferentially found in state I. On the contrary, as state transitions are regulated by the redox state of the PQ pool (Mullineaux and Emlyn-Jones, 2005), it should be expected that any relative loss of PSI excitation will shift the balance toward state II (which would cause an opposite change in the fluorescence spectra than observed).

It has been suggested that PsaF facilitates the association of the PBS to PSI (Fromme et al., 2003) and this has been linked to an elevated APC content in mutants lacking the PsaF subunit, observed especially under low growth light. Here we found that APC is equally increased in $\triangle p s a L$ and in $\triangle F I J L$ (Table 1) and even to a greater extent in low-light-grown cells of both mutants (data not shown). Therefore, we could not discern a particular role of PsaF in the EET from PBS to PSI but the data support the notion that EET is impaired in monomeric PSI. There could be a simple reason for it - supercomplexes of PBSs with trimeric PSI can be more efficient by sharing the PBS among all three PSI RCs - increasing the effective antenna size. Then the increased number of PBSs (APC) in the monomeric PSI strains can be seen as compensatory response for the loss of effective absorption cross-section. However, PBSs can transfer energy to both photosystems (Ashby and Mullineaux, 1999). The PSII:PSI energy partitioning factors have been determined in the range of 50:50 (Tian et al., 2011; Acuña et al., 2018), 60:40 (Mullineaux, 1994) or even higher (Rakhimberdieva et al., 2001). From the Chl ratio in PSII and PSI (4:1) and the fluorescence ratios at 690 and $720 \mathrm{~nm}$ in the low-temperature spectra upon Chls and PBSs excitation (Figure 1), we can estimate that $50-60 \%$ of the energy from PBS is transferred to PSII in WT. Thus, if there is an imbalance of excitation energy between the two photosystems (because of PSI 
monomerization), merely increasing the number of PBSs will exacerbate rather than alleviate it. However, it is known that strains devoid of PBSs or with truncated PBSs rods compensate for the reduced PSII antenna size by increasing the abundance of PSII (Ajlani et al., 1995; Nagarajan et al., 2014; Liberton et al., 2017). Therefore, increasing the number of PBSs while simultaneously reducing their size is a viable strategy to compensate for the effects of PSI monomerization. A similar phenomenon is observed in Anabaena mutants with truncated PBSs, which compensate by increasing the number of PBSs per photosystem (Leganes et al., 2014). Still, the energy distribution in the monomer PSI types is shifted to PSII but to a lesser extent than it would be without the compensatory change in the PBSs.

In conclusion, we have found that the trimeric organization of PSI is advantageous for efficient and balanced EET from PBSs in Synechocystis. It is possible that trimeric PSI better anchors the PBSs and facilitates EET. Alternatively, the reduced EET efficiency to PSI monomers could be because of entropic reasons or simply because monomers are unable to share the same PBS. Regardless of the actual mechanism, the imbalanced excitation is sensed by the cell, which responds by adjusting the PBSs to optimize energy and electron flow. These results add to the existing body of evidence that the PBSs are a remarkably responsive and tuneable light-harvesting antenna system but also provide a hint of the evolutionary advantage of oligomeric PSI in conjunction with PBSs.

\section{Acknowledgements}

We are grateful to Prof. Dario Leister for providing us the $\Delta$ FIJL strain of Synechocystis.

\section{REFERENCES}

Acuña A, Van Alphen P, Van Grondelle R, Van Stokkum I (2018) The phycobilisome terminal emitter transfers its energy with a rate of (20 ps)-1 to photosystem II. Photosynthetica 56: 265274

Ajlani G, Vernotte C, DiMagno L, Haselkorn R (1995) Phycobilisome core mutants of Synechocystis PCC 6803. Biochimica et Biophysica Acta (BBA)-Bioenergetics 1231: 189-196

Akhtar P, Biswas A, Kovacs L, Nelson N, Lambrev PH (2021) Excitation energy transfer kinetics of trimeric, monomeric and subunit-depleted Photosystem I from Synechocystis PCC 6803. Biochem J 478: $1333-1346$

Akhtar P, Biswas A, Petrova N, Zakar T, van Stokkum IHM, Lambrev PH (2020) Time-resolved fluorescence study of excitation energy transfer in the cyanobacterium Anabaena PCC 7120. Photosynthesis Research 144: 247-259

Akita F, Nagao R, Kato K, Nakajima Y, Yokono M, Ueno Y, Suzuki T, Dohmae N, Shen J-R, Akimoto S, Miyazaki N (2020) Structure of a cyanobacterial photosystem I surrounded by octadecameric IsiA antenna proteins. Communications Biology 3: 232

Arteni AA, Ajlani G, Boekema EJ (2009) Structural organisation of phycobilisomes from Synechocystis sp. strain PCC6803 and their interaction with the membrane. Biochimica et Biophysica Acta (BBA) - Bioenergetics 1787: 272-279

Ashby MK, Mullineaux CW (1999) The role of ApcD and ApcF in energy transfer from phycobilisomes to PS I and PS II in a cyanobacterium. Photosynthesis research 61: 169-179

Aspinwall CL, Sarcina M, Mullineaux CW (2004) Phycobilisome mobility in the cyanobacterium Synechococcus sp. PCC7942 is influenced by the trimerisation of photosystem I. Photosynthesis research 79: 179-187 
Calzadilla PI, Kirilovsky D (2020) Revisiting cyanobacterial state transitions. Photochemical \& Photobiological Sciences 19: 585-603

Calzadilla PI, Muzzopappa F, Sétif P, Kirilovsky D (2019) Different roles for ApcD and ApcF in Synechococcus elongatus and Synechocystis sp. PCC 6803 phycobilisomes. Biochimica et Biophysica Acta (BBA)-Bioenergetics 1860: 488-498

Chitnis VP, Chitnis PR (1993) PsaL subunit is required for the formation of photosystem I trimers in the cyanobacterium Synechocystis sp. PCC 6803. FEBS letters 336: 330-334

Dong C, Tang A, Zhao J, Mullineaux CW, Shen G, Bryant DA (2009) ApcD is necessary for efficient energy transfer from phycobilisomes to photosystem I and helps to prevent photoinhibition in the cyanobacterium Synechococcus sp. PCC 7002. Biochimica et Biophysica Acta (BBA)Bioenergetics 1787: 1122-1128

Fromme P, Melkozernov A, Jordan P, Krauss N (2003) Structure and function of photosystem I: interaction with its soluble electron carriers and external antenna systems. FEBS Letters 555: 4044

Fujita Y, Murakami A (1987) Regulation of electron transport composition in cyanobacterial photosynthetic system: stoichiometry among photosystem I and II complexes and their lightharvesting antennae and cytochrome b6/f complex. Plant and cell physiology 28: 1547-1553

Garnier F, Dubacq J-P, Thomas J-C (1994) Evidence for a transient association of new proteins with the Spirulina maxima phycobilisome in relation to light intensity. Plant physiology 106: 747-754

Hippler M, Drepper F, Rochaix J-D, Mühlenhoff U (1999) Insertion of the N-terminal part of PsaF from Chlamydomonas reinhardtii into photosystem I from Synechococcus elongatus enables efficient binding of algal plastocyanin and cytochrome c 6. Journal of Biological Chemistry 274: 4180-4188

Ivanov AG, Krol M, Sveshnikov D, Selstam E, Sandström S, Koochek M, Park Y-I, Vasil'ev S, Bruce D, Öquist G (2006) Iron deficiency in cyanobacteria causes monomerization of photosystem I trimers and reduces the capacity for state transitions and the effective absorption cross section of photosystem I in vivo. Plant physiology 141: 1436-1445

Karapetyan NV, Holzwarth AR, Rögner M (1999) The photosystem I trimer of cyanobacteria: molecular organization, excitation dynamics and physiological significance. FEBS letters 460: $395-400$

Kłodawska K, Kovács L, Várkonyi Z, Kis M, Sozer Ö, Laczkó-Dobos H, Kóbori O, Domonkos I, Strzalka K, Gombos Z, Malec P (2015) Elevated growth temperature can enhance photosystem I trimer formation and affects xanthophyll biosynthesis in cyanobacterium Synechocystis sp. PCC6803 cells. Plant and Cell Physiology 56: 558-571

Kłodawska K, Kovács L, Vladkova R, Rzaska A, Gombos Z, Laczkó-Dobos H, Malec P (2020) Trimeric organization of photosystem I is required to maintain the balanced photosynthetic electron flow in cyanobacterium Synechocystis sp. PCC 6803. Photosynthesis research 143: 251262

Leganes F, Martinez-Granero F, Muñoz-Martín MÁ, Marco E, Jorge A, Carvajal L, Vida T, Gonzalez-Pleiter M, Fernandez-Pinas F (2014) Characterization and responses to environmental cues of a photosynthetic antenna-deficient mutant of the filamentous cyanobacterium Anabaena sp. PCC 7120. Journal of plant physiology 171: 915-926

Li M, Ma J, Li X, Sui S-F (2021) In situ cryo-ET structure of phycobilisome-photosystem II supercomplex from red alga. eLife 10: e69635

Liberton M, Chrisler WB, Nicora CD, Moore RJ, Smith RD, Koppenaal DW, Pakrasi HB, Jacobs JM (2017) Phycobilisome truncation causes widespread proteome changes in Synechocystis sp. PCC 6803. PLoS One 12: e 0173251

Lichtenthaler HK (1987) Chlorophylls and carotenoids: pigments of photosynthetic biomembranes. Methods in enzymology 148: 350-382

Liu H, Blankenship RE (2019) On the interface of light-harvesting antenna complexes and reaction centers in oxygenic photosynthesis. Biochimica et Biophysica Acta (BBA)-Bioenergetics 1860: 148079

Liu H, Zhang H, Niedzwiedzki DM, Prado M, He G, Gross ML, Blankenship RE (2013) Phycobilisomes supply excitations to both photosystems in a megacomplex in cyanobacteria. Science 342: 1104-1107 
MacColl R (1998) Cyanobacterial phycobilisomes. Journal of structural biology 124: 311-334

McConnell MD, Koop R, Vasil'ev S, Bruce D (2002) Regulation of the distribution of chlorophyll and phycobilin-absorbed excitation energy in cyanobacteria. A structure-based model for the light state transition. Plant physiology 130: 1201-1212

Mullineaux CW (1994) Excitation energy transfer from phycobilisomes to photosystem I in a cyanobacterial mutant lacking photosystem II. Biochimica et Biophysica Acta (BBA) Bioenergetics 1184: 71-77

Mullineaux CW, Emlyn-Jones D (2005) State transitions: an example of acclimation to low-light stress. Journal of Experimental Botany 56: 389-393

Mullineaux CW, Holzwarth AR (1991) Kinetics of excitation energy transfer in the cyanobacterial phycobilisome-photosystem II complex. Biochimica et Biophysica Acta (BBA) - Bioenergetics 1098: $68-78$

Murakami A, Fujita Y (1991) Regulation of photosystem stoichiometry in the photosynthetic system of the cyanophyte Synechocystis PCC 6714 in response to light-intensity. Plant and cell physiology 32: 223-230

Nagarajan A, Page LE, Liberton M, Pakrasi HB (2014) Consequences of decreased light harvesting capability on photosystem II function in Synechocystis sp. PCC 6803. Life 4: 903-914

Nomsawai P, de Marsac NT, Thomas JC, Tanticharoen M, Cheevadhanarak S (1999) Light regulation of phycobilisome structure and gene expression in Spirulina platensis C1 (Arthrospira sp. PCC 9438). Plant and cell physiology 40: 1194-1202

Rakhimberdieva MG, Boichenko VA, Karapetyan NV, Stadnichuk IN (2001) Interaction of phycobilisomes with photosystem II dimers and photosystem I monomers and trimers in the cyanobacterium Spirulina platensis. Biochemistry 40: 15780-15788

Raps S, Kycia JH, Ledbetter MC, Siegelman HW (1985) Light intensity adaptation and phycobilisome composition of Microcystis aeruginosa. Plant physiology 79: 983-987

Rast A, Schaffer M, Albert S, Wan W, Pfeffer S, Beck F, Plitzko JM, Nickelsen J, Engel BD (2019) Biogenic regions of cyanobacterial thylakoids form contact sites with the plasma membrane. Nature Plants 5: 436-446

Salomon E, Bar-Eyal L, Sharon S, Keren N (2013) Balancing photosynthetic electron flow is critical for cyanobacterial acclimation to nitrogen limitation. Biochimica et Biophysica Acta (BBA)Bioenergetics 1827: 340-347

Salomon E, Keren N (2011) Manganese limitation induces changes in the activity and in the organization of photosynthetic complexes in the cyanobacterium Synechocystis sp. strain PCC 6803. Plant physiology 155: 571-579

Samson G, Herbert SK, Fork DC, Laudenbach DE (1994) Acclimation of the photosynthetic apparatus to growth irradiance in a mutant strain of Synechococcus lacking iron superoxide dismutase. Plant physiology 105: 287-294

Sandström Å, Gillbro T, Sundström V, Wendler J, Holzwarth AR (1988) Picosecond study of energy transfer within 18-S particles of AN 112 (a mutant of Synechococcus 6301) phycobilisomes. Biochimica et Biophysica Acta (BBA)-Bioenergetics 933: 54-64

Sarcina M, Tobin MJ, Mullineaux CW (2001) Diffusion of phycobilisomes on the thylakoid membranes of the cyanobacterium Synechococcus 7942: effects of phycobilisome size, temperature, and membrane lipid composition. Journal of Biological Chemistry 276: 46830 46834

Schluchter WM, Shen G, Zhao J, Bryant DA (1996) Characterization of psal and psaL mutants of Synechococcus sp. strain PCC 7002: a new model for state transitions in cyanobacteria. Photochemistry and Photobiology 64: 53-66

Şener MK, Park S, Lu D, Damjanović A, Ritz T, Fromme P, Schulten K (2004) Excitation migration in trimeric cyanobacterial photosystem I. The Journal of chemical physics 120: 11183-11195

Stadnichuk I, Krasilnikov P, Zlenko D (2015) Cyanobacterial phycobilisomes and phycobiliproteins. Microbiology 84: 101-111

Tian L, van Stokkum IH, Koehorst RB, Jongerius A, Kirilovsky D, van Amerongen H (2011) Site, rate, and mechanism of photoprotective quenching in cyanobacteria. Journal of the American Chemical Society 133: 18304-18311 
Ueno Y, Aikawa S, Niwa K, Abe T, Murakami A, Kondo A, Akimoto S (2017) Variety in excitation energy transfer processes from phycobilisomes to photosystems I and II. Photosynthesis research 133: $235-243$

Zavřel T, Chmelík D, Sinetova MA, Červený J (2018) Spectrophotometric Determination of Phycobiliprotein Content in Cyanobacterium Synechocystis. JoVE: e58076 transfer among the rod-core complex from phycobilisome of Anabaena variabilis by time resolved fluorescence emission and anisotropy spectra. Biochimica et Biophysica Acta (BBA)Bioenergetics 1320: 285-296

Zheng L, Zheng Z, Li X, Wang G, Zhang K, Wei P, Zhao J, Gao N (2021) Structural insight into the mechanism of energy transfer in cyanobacterial phycobilisomes. Nature Communications 12: $1-11$

Zlenko D, Krasilnikov PM, Stadnichuk IN (2016) Structural modeling of the phycobilisome core and its association with the photosystems. Photosynthesis research 130: 347-356

Zlenko DV, Elanskaya IV, Lukashev EP, Bolychevtseva YV, Suzina NE, Pojidaeva ES, Kononova IA, Loktyushkin AV, Stadnichuk IN (2019) Role of the PB-loop in ApcE and phycobilisome core function in cyanobacterium Synechocystis sp. PCC 6803. Biochimica et Biophysica Acta

604 (BBA)-Bioenergetics 1860: 155-166 